\title{
Altered vascular permeability and early onset of experimental autoimmune encephalomyelitis in PECAM-1-deficient mice
}

\author{
Donnasue Graesser, ${ }^{1}$ Anna Solowiej, ${ }^{1}$ Monika Bruckner, ${ }^{2}$ Emily Osterweil,, ${ }^{1}$ Amy Juedes, ${ }^{3}$ \\ Sandra Davis, ${ }^{1}$ Nancy H. Ruddle, ${ }^{3}$ Britta Engelhardt, ${ }^{2}$ and Joseph A. Madri ${ }^{1}$ \\ ${ }^{1}$ Department of Pathology, Yale University School of Medicine, New Haven, Connecticut, USA \\ ${ }^{2}$ Max-Planck-Institute, Bad Nauheim, Germany \\ ${ }^{3}$ Department of Immunobiology, Yale University School of Medicine, New Haven, Connecticut, USA \\ Address correspondence to: Joseph A. Madri, Department of Pathology, \\ Yale University School of Medicine, 310 Cedar Street, New Haven, Connecticut 06520, USA. \\ Phone: (203) 785-2763; Fax: (203) 785-7373; E-mail: joseph.madri@yale.edu.
}

Received for publication June 21, 2001, and accepted in revised form December 27, 2001.

Platelet/endothelial cell adhesion molecule-1 (PECAM-1, CD31), a 130-kDa glycoprotein member of the Ig superfamily of transmembrane proteins, is expressed on endothelial cells, platelets, and subsets of leukocytes. It functions as a cell adhesion molecule as well as a scaffolding molecule capable of modulating cellular signaling pathways. In this study, using PECAM-1-deficient (KO) mice, as well as cells derived from these mice, we demonstrate that the absence of PECAM- 1 expression is associated with an early onset of clinical symptoms during experimental autoimmune encephalomyelitis (EAE), a mouse model for the human autoimmune disease multiple sclerosis. During EAE, mononuclear cell extravasation and infiltration of the CNS occur at earlier time points in PECAM-KO mice than in wild-type mice. In vitro, $T$ lymphocyte transendothelial migration across PECAM-KO endothelial cells is enhanced, regardless of expression of PECAM-1 on transmigrating T cells. Additionally, cultured PECAM-KO endothelial cells exhibit prolonged permeability changes in response to histamine treatment compared with PECAM-1-reconstituted endothelial cells. Lastly, we demonstrate an exaggerated and prolonged CNS vascular permeability during the development of EAE and a delay in restoration of dermal vascular integrity following histamine challenge in PECAM-KO mice.

J. Clin. Invest. 109:383-392 (2002). DOI:10.1172/JCI200213595.

\section{Introduction}

Platelet/endothelial cell adhesion molecule-1 (PECAM1 ) is a $130-\mathrm{kDa}$ glycoprotein belonging to the Ig superfamily of cell adhesion molecules (1). PECAM-1 is widely expressed on endothelial cells $(2,3)$, circulating platelets, monocytes, granulocytes, subsets of lymphocytes, and bone marrow hematopoietic cells (4-7). On continuous endothelial cell monolayers, PECAM-1 expression is concentrated at areas of cell-cell contact $(2,3)$. As a cell adhesion molecule, PECAM- 1 is capable of mediating homophilic binding (8-10) as well as heterophilic binding $(11,12)$.

PECAM-1 is thought to be involved in the transendothelial migration of leukocytes during their extravasation to sites of inflammation. It is an ideal candidate molecule for this role due to (a) its expression on most circulating leukocytes as well as the endothelial cells lining the vasculature; (b) its localization at endothelial cell-cell junctions, the site of leukocyte diapedesis; and (c) its ability to mediate homophilic as well as both heterophilic and heterotypic adhesion. In vitro studies using PECAM-1-blocking reagents have demonstrated that PECAM-1 plays a role in transmigration of leukocytes in vitro (13-15) and in animal models of inflammation (16-19). However, it has also been demonstrated that the involvement of PECAM-1 is selective for leukocyte migration elicited by specific inflammatory stimuli (18). The expression of PECAM-1 by transmigrating leukocytes does not appear to be obligatory, as anti-PECAM blocking antibodies do not inhibit transmigration completely. In addition, a T cell population depleted of PECAM- $1^{+}$ cells was able to migrate across human umbilical vein endothelial cell monolayers (20). In PECAM-1-deficient mice (21) there is a transient delay in polymorphonuclear leukocyte trafficking across the subendothelial basal lamina during acute inflammation, but normal numbers of migrated cells are recovered from the inflammatory site. Additionally, PECAM-1-deficient (PECAM-KO) mice are able to mount a normal cutaneous hypersensitivity response (21).

PECAM-1 may also play an indirect role in leukocyte extravasation. Numerous cellular "activation" stimuli induce the phosphorylation of the PECAM-1 cytoplasmic domain (22-26), which in turn can interact with phosphatases (27-29) and kinases $(26,30)$ to mediate a number of intracellular signaling events. Engagement of PECAM- 1 signals increases in $\beta 1$ and $\beta 2$ integrin affinity $(15,31-34)$, leading to firm adhesion of circulating leukocytes to vascular endothelium during 
extravasation (35). Both migration of leukocytes and signaling of effector functions of those cells are essential components of an immune response, and there is a growing body of evidence to suggest that PECAM- 1 is involved in modulating cellular behavior of leukocytes $(25,34,36,37)$.

Here, we examine the contribution of PECAM-1 expression on endothelium and antigen-specific $\mathrm{T}$ cells to a specific model of inflammation, experimental autoimmune encephalomyelitis (EAE). EAE is an autoimmune inflammatory disease of the CNS in mice, used as a model to study the human demyelinating disease multiple sclerosis (38). Progression of EAE is regulated at many points during the course of disease, including activation of autoimmune $T$ cells, $T$ cell adhesion to blood vessel endothelium, transmigration across the blood-brain barrier, establishment of an autoimmune response in the CNS, recruitment of inflammatory cells to the CNS, and maintenance of CNS inflammation. Adhesion or signaling mediated by PECAM-1 could potentially play a role in any of these processes. Administration of PECAM- 1 antibodies had no effect on the course of EAE in a rat model (39). However, the generation of the PECAM-KO mouse (21) along with the development of the myelin oligodendrocyte glycoprotein (MOG) immunization model for chronic EAE in C57BL/6 mice (40) provided us with a model in which to study the clinical progression of EAE in the complete absence of PECAM-1. In this report, we demonstrate that the absence of PECAM- 1 expression in mice results in an early onset of clinical symptoms during EAE, associated with an increased leukocyte migration across the blood-brain barrier. We also demonstrate an impaired ability to restore vascular integrity after alteration of the endothelium in PECAM-KO mice or of cultured PECAM-KO endothelial cells. The resulting increased and prolonged vascular permeability could potentially lead to enhanced transendothelial migration of leukocytes and early onset of CNS inflammation during EAE.

\section{Methods}

Antibodies and reagents. Monoclonal anti-PECAM-1 (MEC13.3), VE-cadherin (11D4.1), CD4 (RM4-5), CD8 (53-6.7), Mac-1 (M1/70), B220 (RA3-6B2), and Gr-1 (Ly-6G, RB6-8 C5) were purchased from PharMingen (San Diego, California, USA). Fluorophore-conjugated secondary antibodies were purchased from Jackson ImmunoResearch Laboratories Inc. (West Grove, Pennsylvania, USA). Rat anti-mouse $\mathrm{mAb}$ 's used at the Max-Planck-Institute were purified from serum-free hybridoma supernatants. Anti-PECAM-1 (MEC13.3 or GC.51), anti-ICAM-1 (25ZC7), anti-ICAM-2 (3C4), anti-VCAM-1 (9B5), and anti-human CD44 (9B5) as an isotype-matched control were previously described $(41,42)$. MOG peptide 35-55 (MEVGWYRSPFSRVVHLYRNGK) of rat origin was synthesized by the W.M. Keck Biotechnology Resource Center at Yale University.
Cells and cell culture

Antigen-specific T cell lines. MOG-specific T cells were derived from C57BL/6 mice immunized with purified MOG peptide 35-55 (300 $\mu$ g per mouse) emulsified 1:1 in CFA (Difco Laboratories, Detroit, Michigan, USA). Ten days later, cells were harvested from draining lymph nodes and cultured. Expression of PECAM-1 and $T$ cell markers was analyzed by flow cytometry. The derivation and maintenance of the proteolipid protein-specific (PLP-specific) T cell lines were described in detail previously $(42,43)$.

Microvascular endothelial cells. Microvascular endothelial cells used in Figure 3 were harvested and cultured according to the method described by Plendl et al. (41). Endothelial cells were isolated by FACS sorting on PECAM-1/VE-cadherin double-positive cells (wild-type [WT]) or VE-cadherin-positive cells (KO) and used for experiments from passages 2 to 4 .

Endothelioma cell lines. PECAM-KO endothelioma cell lines bEnd.PECAM-1.2, mlEnd.PECAM-1.1, and luEnd.PECAM-1.1 were established by retroviral transduction of primary endothelial cell culture with the polyoma virus middle T-oncogene as described $(44,45)$. The endothelioma cell lines retained endothelial morphology and showed contact inhibition upon confluence. The wild-type line bEnd.WT derived from mouse brain endothelium was used as a control because, with the exception of PECAM-1, the cell surface expression levels of adhesion molecules, most importantly ICAM-1 and ICAM-2, were similar to those of the PECAM-1-deficient endothelioma cell lines as determined by FACS analysis. Endothelioma cells were used for experiments from passages 8 to 25 .

Retroviral infection of endothelioma cells. A full-length murine PECAM-1 cDNA fragment was isolated from a pcDNA3 plasmid clone (45) (courtesy of Beat Imhof, University of Geneva, Geneva, Switzerland) and ligated into pSL1190 (Pharmacia Biotech, Cambridge, United Kingdom). The full-length PECAM-1 fragment was released and ligated into pBABE puromycin (46). The resulting construct was transfected into the retrovirus packaging cell line GP+E86, and stable clones were selected in the presence of puromycin as described before (44). Supernatant of GP+E86 PECAM-1 transfectants supplemented with $8 \mu \mathrm{g} / \mathrm{ml}$ Polybrene (SigmaAldrich Co., St. Louis, Missouri, USA) was used to transduce the PECAM-1-deficient endotheliomas with full length PECAM-1. Clones were grown to passage 5 and expression of PECAM-1 in retransfected cells was analyzed by FACS analysis and immunofluorescence. The PECAM-1-deficient endothelioma line luEnd.PECAM-1.1-clone 9 and its respective retransfected line luEnd.PPA2, termed PECAM-KO and PECAM-RC (reconstituted with PECAM-1), respectively, were used in this study.

Mice. C57BL/6 WT mice were purchased from Charles River Laboratories (Wilmington, Massachusetts, USA). PECAM-1-deficient (PECAM-KO) mice were generated and analyzed as described by Duncan 
et al. (21). Before breeding pairs were sent to Yale University, the PECAM-1-deficient mice were backcrossed onto $\mathrm{C} 57 \mathrm{BL} / 6$ eight times. At Yale University the PECAM-KO mice were backcrossed onto C57BL/ 6 several more times before experimentation was begun.

Hematopoietic precursor engraftment of WT and PECAM-KO mice was performed as described (47).

Induction of EAE with MOG peptide. EAE was induced using a MOG immunization protocol in C57BL/6 mice, according to the procedure previously described (40). The mice were monitored daily and graded for clinical symptoms of EAE. Some mice were sacrificed at indicated times, and CNS tissues were prepared for slides. This MOG immunization protocol in C57BL/6 mice induces chronic, nonremitting EAE clinical symptoms (40).

Adoptive transfer of EAE with MOG peptide-specific T cells derived from WT and PECAM-KO C57BL/6 mice. EAE was induced using an adoptive transfer protocol in C57BL/6 mice according to the previously published procedure (40). The mice were monitored daily and graded for clinical symptoms of EAE $(48,49)$.

Isolation oflenkocytes from CNS. To isolate lymphocytes and monocytes, CNS tissue was dissected, homogenized, incubated with $1 \mathrm{mg} / \mathrm{ml}$ collagenase II (SigmaAldrich Co.), and run over a gradient of PBS-buffered Percoll (Amersham Pharmacia Biotech, Piscataway, New Jersey, USA). Isolated cells were analyzed by flow cytometry. For detection of intracellular cytokine production, cells were isolated as above and cultured with the protein transport inhibitors GolgiStop $(0.67 \mu \mathrm{l} / \mathrm{ml}$; for IFN- $\gamma$ ) or GolgiPlug $(1 \mu \mathrm{l} / \mathrm{ml}$; for TNF- $\alpha)$. Cells were then resuspended and stained for CD4 or Mac-1 as above. Next, cells were fixed and permeabilized with Cytofix/Cytoperm, stained for IFN- $\gamma$ or TNF- $\alpha$, and analyzed by flow cytometry. GolgiStop, GolgiPlug, and Cytofix/Cytoperm were purchased from PharMingen.

$T$ cell priming assay. WT and PECAM-KO mice were immunized subcutaneously in the tail with $50 \mu \mathrm{l}$ of a 1:1 MOG/CFA emulsion at the same concentrations as used for EAE studies. After 10 days, cells were harvested from draining lymph nodes. Triplicate wells of a 96-well microtiter plate were prepared with $2 \times 10^{5} \mathrm{lymph}$ node cells in $200 \mu \mathrm{l}$ of Bruff's medium containing $10 \%$ FCS, and varying concentrations of MOG peptide antigen $(12.5-50 \mu \mathrm{g} / \mathrm{ml})$. After 48 hours in culture, each sample was pulsed with $1 \mu \mathrm{Ci}{ }^{3} \mathrm{H}$-thymidine, incubated for an additional 16 hours, and collected using a Skatron 12 Channel Combi Cell Harvester (Skatron Instruments, Sterling, Virginia, USA), and proliferative response to MOG peptide antigen was measured in cpm of $\beta$-radiation incorporated into the proliferating cell cultures.

$T$ cell transendothelial migration assays. A Biocoat tissue culture insert system was used (Becton Dickinson Labware, Franklin Lakes, New Jersey, USA) to measure in vitro transmigration of $\mathrm{T}$ cells through monolayers of WT and PECAM-KO microvascular endothelial cells (Figure 3), as previously described $(48,49)$. Transmigration assays in Figure 4 were performed exactly as previously described $(42,44)$.
In vitro endothelial permeability assays. To measure permeability changes of endothelial cells in vitro, endothelioma lines were cultured to confluence on Biocoat tissue culture inserts placed in tissue culture wells (Becton Dickinson Labware). Culture media were replaced with colorless HBSS. In control samples, $20 \mu \mathrm{l}$ of $0.5 \%$ (wt $/ \mathrm{vol}$ ) Evans blue dye was added to the upper compartment to ensure that the endothelial layers were impermeable to this dye. Experimental wells were treated with histamine $\left(3.2 \times 10^{-5} \mathrm{M}\right.$; Sigma-Aldrich Co.), and $20 \mu \mathrm{l}$ dye was added to the upper compartments at various time points following histamine exposure. The liquid was collected from the lower wells after 2 minutes and measured by spectrophotometric absorbance at $650 \mathrm{~nm}$.

In vivo endothelial permeability assays. At various days following EAE induction, mice were injected retro-orbitally with $0.5 \%$ (wt $/ \mathrm{vol})$ Evans blue dye in PBS $(4 \mu \mathrm{l} / \mathrm{g}$ of body weight) (50). After 1 hour, mice were anesthetized and perfused via the left ventricle with cold PBS, and brains were removed. Dye was extracted from the CNS tissue for 3 days in formamide ( $5 \mu \mathrm{l} / \mathrm{mg}$ of tissue) at room temperature. Extracted dye concentration was determined by measuring the absorbance at $650 \mathrm{~nm}$. The blood-brain barrier permeability index (PI) was calculated by dividing the OD value for each sample by the average OD value of tissue from three mice that had not received Evans blue dye. Skin vessel permeability of each mouse was determined similarly in Figure 6b.

In Figure $6 c$, skin vessel permeability was determined following intradermal histamine challenge, according to a modification of the Miles assay (51). Mice were given an intradermal injection of histamine ( $3 \mu \mathrm{g}$ in $50 \mu \mathrm{l} \mathrm{PBS})$ in one flank and a 50- $\mu$ injection of PBS in the opposite flank. At indicated time points after histamine injection, Evans blue dye was injected retro-orbitally as above. Mice were sacrificed 5 minutes after dye injection, and skin was harvested. Dye was extracted in formamide as above, and absorbance at $650 \mathrm{~nm}$ was measured. The PI for each mouse was determined by dividing the OD value obtained from the histamine-injected flank skin by the value obtained from the PBS-injected flank skin.

In Figure 6d, mice were first injected retro-orbitally with Evans blue as above. After 45 minutes, histamine and PBS were injected intradermally as above. At 5 and 15 minutes after histamine injection, mice were sacrificed, skin was dissected, dye was extracted in formamide $(10 \mu \mathrm{l} / \mathrm{mg}$ of tissue) at $56^{\circ} \mathrm{C}$ for 24 hours, and PI was calculated as above.

Statistics. Results were analyzed using two-way ANOVA, with two between-group factors (SAS 8.2 software; SAS Institute Inc., Cary, North Carolina, USA) (Figures 3 and 4), or using N-way ANOVA and an allpairwise multiple comparison procedure (StudentNewman-Keuls method) (SPSS Science, Chicago, Illinois, USA) (Figures 1, 2, 5, and 6).

\section{Results}

PECAM-KO mice exbibit early onset of EAE and early inflammatory cell migration into CNS. To examine the role of PECAM-1 in MOG-induced EAE, C57BL/ 6 WT 

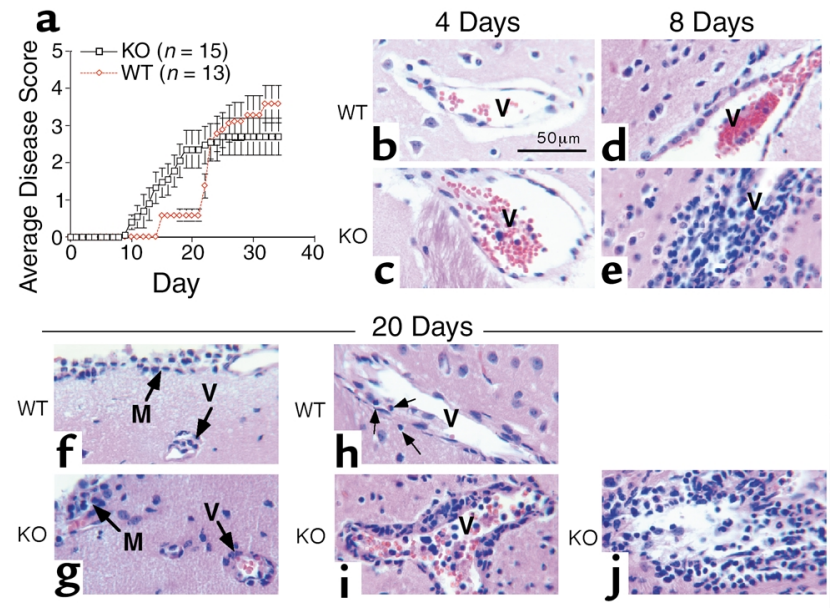

\section{Figure 1}

PECAM-KO mice exhibit early incidence of EAE and earlier mononuclear cell infiltration of CNS parenchyma. (a) Average disease scores of WT and PECAM-KO mice calculated daily following induction of disease with MOG peptide. Disease symptoms manifested earlier in the PECAM-KO mice than in WT mice. (b-j) Representative hematoxylin and eosin-stained sections of cerebral cortex taken from the brains of WT ( $\mathbf{b}, \mathbf{d}, \mathbf{f}$, and $\mathbf{h})$ and PECAM-KO mice (c, e, $\mathbf{g}, \mathbf{i}$, and $\mathbf{j}$ ) at 4 days (b and $\mathbf{c}$ ), 8 days ( $\mathbf{d}$ and $\mathbf{e}$ ), and 20 days $(\mathbf{f}-\mathbf{j})$ following EAE induction. PECAM-KO animals exhibited more intense perivascular (i) and parenchymal (j) infiltrates than their WT counterparts (h), which correlated with the difference in clinical disease scores noted at this time (a). Arrows indicate perivascular mononuclear cells. V, vessel; $\mathrm{M}$, meninges. Scale bar $=50 \mu \mathrm{m}$.

and PECAM-KO mice were immunized with MOG peptide antigen and monitored daily for clinical symptoms of EAE (Figure 1a). The PECAM-KO mice were susceptible to MOG-induced EAE and developed clinical disease symptoms significantly earlier than did WT mice. The differences in the mean values among the groups (WT and $\mathrm{KO}$ ) are greater than would be expected by chance $\left(F=24.17, P<2.23 \times 10^{-30}\right)$. An allpairwise multiple comparison procedure resulted in significant differences between the two groups from days 12 through 22 . By day 24 the average severity of disease scores was comparable between WT and PECAM-KO mice. Both groups of mice exhibited chronic disease, which is typical of MOG-induced EAE in the C57BL/6 model (40).
To determine the extent of inflammatory cell trafficking into the CNS parenchyma, brain tissues of WT and PECAM-KO mice were examined (Figure 1, b-j). In comparison to WT mice, perivascular mononuclear cell infiltrates were noted earlier in PECAM-KO tissues. Specifically, at 4 days after immunization neither WT nor PECAM-KO mice exhibited appreciable perivascular or parenchymal invasion of inflammatory cells into the CNS (Figure 1, b and c). At day 8, the brains of WT mice were mostly devoid of inflammatory infiltrates, while substantial perivascular cuffing of mononuclear cells was observed in the brains of PECAM-KO mice (Figure 1, d and e). By day 20, brains of both WT and PECAM-KO mice exhibited modest meningeal and submeningeal perivascular infiltrates (Figure 1, $\mathrm{f}$ and g). However, brains of PECAM-KO mice exhibited more robust invasion around larger, deeper vessels compared with WT mice, corresponding to significantly higher disease severity scores (Figure 1, h and i). Lytic foci were also apparent in brains of PECAM-KO mice (Figure 1j). At later time points, when the average clinical disease scores were similar for both groups of mice (days 24-30), the inflammatory infiltrates were similar to those illustrated in Figure 1, $i$ and $j$, and were indistinguishable from each other (data not shown).

Leukocytes were isolated from the CNS of WT and PECAM-KO mice in the early stages of EAE to determine the composition of invading cells. In comparison to WT mice, increased numbers of total leukocytes, $\mathrm{CD}^{+}$and $\mathrm{CD}^{+} \mathrm{T}$ cells, $\mathrm{B}$ cells, total macrophages, TNF- $\alpha-$ producing (activated) macrophages, and neutrophils were present in the CNS of PECAM-KO mice (Table 1). Increases in MOG-specific $\mathrm{CD}^{+} \mathrm{T}$ cells were also seen earlier and in greater numbers in the CNS of PECAM-KO mice (data not shown).

The increased presence of inflammatory cells in the brain tissue of PECAM-KO mice may be due to either an enhanced initial immune response to antigen or an increased ability of inflammatory cells to transmigrate across the blood-brain barrier. Because PECAM-1 possesses the ability to modulate signal transduction, the initial response of MOG-specific $T$ cells to antigen may be increased. Following immunization with MOG peptide, cells were isolated from draining lymph nodes. There is no significant difference between WT and

Table 1

PECAM-KO mice have more leukocytes in the CNS early in EAE

\begin{tabular}{|c|c|c|c|c|c|c|c|}
\hline Group $^{A}$ & $\begin{array}{l}\text { Total CNS } \\
\text { leukocytes }\end{array}$ & $\mathrm{CD}^{+}$ & $\mathrm{CD}^{+}$ & Macrophages & B cells & Neutrophils & $\begin{array}{c}\text { MOG-activated } \\
\text { macrophages }\end{array}$ \\
\hline $\begin{array}{l}\text { WT } \\
\text { SEM }\end{array}$ & $\begin{array}{l}1.47 \times 10^{6} \\
0.26 \times 10^{6}\end{array}$ & $\begin{array}{l}0.75 \times 10^{5} \\
0.49 \times 10^{5}\end{array}$ & $\begin{array}{l}0.33 \times 10^{5} \\
0.79 \times 10^{4}\end{array}$ & $\begin{array}{l}1.76 \times 10^{5} \\
0.37 \times 10^{5}\end{array}$ & $\begin{array}{l}1.29 \times 10^{5} \\
0.62 \times 10^{5}\end{array}$ & $\begin{array}{l}1.00 \times 10^{5} \\
0.28 \times 10^{5}\end{array}$ & $\begin{array}{l}2.24 \times 10^{5} \\
0.73 \times 10^{5}\end{array}$ \\
\hline $\begin{array}{l}\text { PECAM-KO } \\
\text { SEM }\end{array}$ & $\begin{array}{l}4.17 \times 10^{6} \\
0.81 \times 10^{6}\end{array}$ & $\begin{array}{l}5.45 \times 10^{5} \\
0.55 \times 10^{5}\end{array}$ & $\begin{array}{l}2.96 \times 10^{5} \\
1.64 \times 10^{5}\end{array}$ & $\begin{array}{l}8.51 \times 10^{5} \\
2.31 \times 10^{5}\end{array}$ & $\begin{array}{l}2.81 \times 10^{5} \\
0.50 \times 10^{5}\end{array}$ & $\begin{array}{l}3.30 \times 10^{5} \\
0.67 \times 10^{5}\end{array}$ & $\begin{array}{l}1.17 \times 10^{6} \\
0.27 \times 10^{4}\end{array}$ \\
\hline Significance & $P<0.03$ & $P<0.003$ & $P<0.18$ & $P<0.04$ & $P<0.13$ & & \\
\hline
\end{tabular}

Inflammatory cell infiltration of the CNS during EAE is increased in PECAM-KO mice. Shown are numbers of leukocytes isolated from the CNS (brain plus spinal cord) of WT and PECAM-KO mice, during the early stages of EAE. AEach number is an average of three experiments, except for neutrophils and activated macrophages, which are averages of two experiments. 
PECAM-KO lymph node cells in their ability to proliferate in response to a range of concentrations of MOG peptide antigen (Figure 2a). In addition, we have demonstrated, using adoptive transfer techniques (47, 48), an earlier onset of EAE in KO mice than in WT mice, regardless of whether the mice were injected intravenously with antigen-specific (MOG) WT or PECAM-KO T lymphocytes (Figure $2 \mathrm{~b}$ ). The differences in the mean values among the animal groups (WT and $\mathrm{KO}$ mice) are greater than would be expected by chance $\left(F=3.85, P<7.1 \times 10^{-9}\right)$. An all-pairwise multiple comparison procedure resulted in significant differences between the two groups from days 13 through 16. No differences were noted in KO mice given WT or KO MOG-specific T lymphocytes. Further, using WT mice lethally irradiated and engrafted with PECAM-KO marrow and $\mathrm{KO}$ mice lethally irradiated and engrafted with WT marrow (47), we demonstrated an earlier disease onset of EAE in the $\mathrm{KO}$ mice following direct immunization with MOG peptides (Figure 2c). The differences in the mean values among the marrow recipient groups (WT mice engrafted with KO marrow and KO mice engrafted with WT marrow) are greater than would be expected by chance $\left(F=7.9, P<3.1 \times 10^{-22}\right)$. An all-pairwise multiple comparison procedure resulted in significant differences between the two groups from days 13 through 17 . These studies support the concept that while EAE is a T cell-mediated disease process, it is dependent, in part, upon the presence or absence of endothelial PECAM-1. Therefore, we investigated a second possibility that transendothelial migration of immune system cells may play a role in the early onset of EAE in the PECAM-KO mice.

Endothelial cells derived from PECAM-KO mice support enhanced in vitro transendothelial migration of T cells. We performed in vitro transmigration assays using low-passage endothelial cells and MOG-specific $T$ cells derived from WT and PECAM-KO mice (Figure 3, a-c). Interestingly, endothelial cells derived from PECAM-KO mice supported a significantly higher level of $\mathrm{T}$ cell transmigration $(\sim 42 \%)$ compared with WT endothelial cells $(\sim 31 \%)$, regardless of expression of PECAM-1 on the T cells. Two-way ANOVA, with two between-group factors, revealed a significant main effect for the endothelial cell type (WT versus $\mathrm{KO}$ ) $\left[F_{1,28}=113.02, P<0.001\right]$. The main effect for $\mathrm{T}$ cell type (WT versus $\mathrm{KO}$ ) proved to be insignificant, $\left[F_{1,28}=0.04, P=0.85\right]$, as did the interaction between endothelial cell type (WT versus KO) and $\mathrm{T}$ cell type (WT versus $\mathrm{KO}$ ) $\left[F_{1,28}=0.10, P=0.76\right]$.

In addition, we performed adhesion and transmigration assays using immortalized endothelioma cell lines derived from brain microvasculature of WT and PECAM-KO mice (Figure 4a) and lung microvasculature of PECAM-KO mice, and the same cells reconstituted with PECAM-1 (PECAM-RC) (Figure 4b). We found the level of adhesion of MOG-specific T cells derived from WT (Figure 4c) and PECAM-KO (Figure 4 e) animals to these four endothelioma lines indistinguishable (Figure 4, c and e). Analyses using two-way
ANOVA revealed no significant differences between groups. In contrast, migration of $\mathrm{T}$ cells across PECAM-KO endotheliomas derived from brain (bEnd.PECAM-1.2) and lung (luEnd.PECAM-1.1) was significantly increased in comparison with transmigration across WT endothelioma monolayers derived from brain (bEnd.WT) and KO endothelioma monolayers derived from lung (luEnd.PECAM-1.1) that were transfected and stably expressing PECAM-1 (PECAM-RC) (Figure 4, $d$ and f). These endothelioma
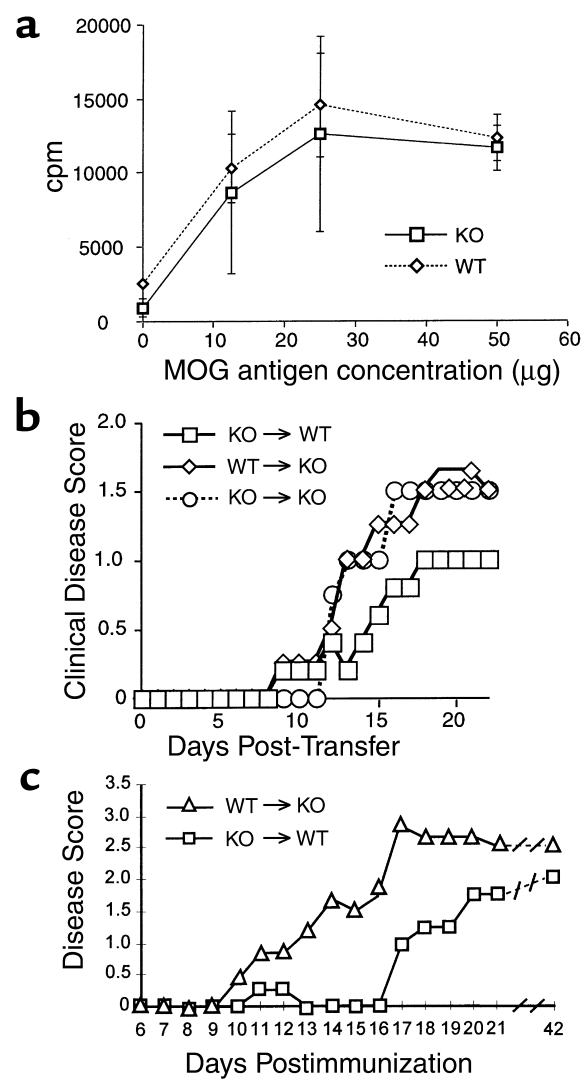

\section{Figure 2}

Antigen-specific T lymphocytes derived from PECAM-KO and WT mice are indistinguishable in in vitro priming assays (a) and in vivo adoptive transfer (b) and marrow engraftment assays (c). (a) In vitro priming assay illustrating that the proliferation of in vivo MOG peptide-primed T lymphocytes harvested from the draining lymph nodes of PECAM-KO and WT mice is indistinguishable. $n=5$. Vertical lines represent standard deviations. (b) Adoptive transfer study illustrating that the time of onset of EAE correlates with the presence or absence of endothelial PECAM-1. KO $\rightarrow$ WT = antigen-specific PECAM-1-deficient $T$ lymphocytes adoptively transferred into a WT recipient. WT $\rightarrow \mathrm{KO}=$ antigen-specific PECAM-1-positive T lymphocytes adoptively transferred into a PECAM-1-deficient recipient. $\mathrm{KO} \rightarrow \mathrm{KO}=$ antigen-specific PECAM-1-deficient $\mathrm{T}$ lymphocytes adoptively transferred into a PECAM-1-deficient recipient. $n=4$. (c) PECAM-KO mice engrafted with WT marrow precursors $(\mathrm{WT} \rightarrow \mathrm{KO}$ ), but not WT mice engrafted with PECAM-KO marrow precursors $(\mathrm{KO} \rightarrow \mathrm{WT})$, exhibit an early incidence of EAE. This study also illustrates that the time of onset of EAE correlates with the presence or absence of endothelial PECAM-1. This graph represents an average of one of three independent experiments, each having groups of three WT $\rightarrow \mathrm{KO}$ and $\mathrm{KO} \rightarrow \mathrm{WT}$ mice. 


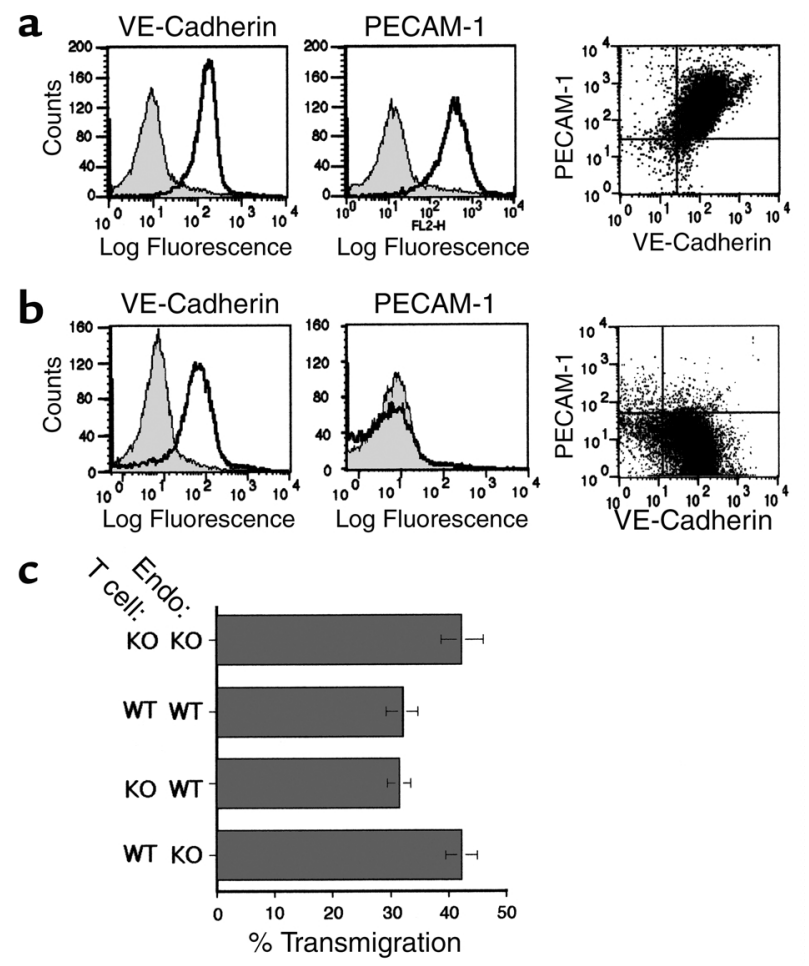

Figure 3

Endothelial cells derived from PECAM-KO mice support increased transmigration of MOG-specific T cells. ( $\mathbf{a}$ and $\mathbf{b})$ FACS analyses of microvascular endothelial cells derived from WT (a; PECAM- $1^{+}$, VE-cadherin $\left.{ }^{+}\right)$and KO (b; PECAM-1-, VE-cadherin $\left.{ }^{+}\right)$mice. These cells were used for in vitro transendothelial migration assays of activated MOG-specific T cells. (c) PECAM-KO endothelial cells supported a $31 \%$ increase in transmigration compared with WT endothelial cells $(P \leq 0.0001, n=8)$, regardless of expression of PECAM- 1 by the $T$ cells. Endo, endothelium.

lines express equivalent levels of VE-cadherin, ICAM-1, ICAM-2 (not shown), and VCAM-1 (not shown), but only WT and PECAM-RC cells express PECAM-1 (Figure 4, a and b). In these transmigration assays the percent transmigration was also noted to be dependent upon PECAM-1 expression on the MOGspecific T lymphocyte clones, with the WT T lymphocytes exhibiting a greater percent transmigration than the KO T lymphocytes (Figure 4, compare d with $\mathrm{f}$ ). Two-way ANOVA, with two between-group factors, revealed a significant main effect for endothelial cell type (brain WT versus $\mathrm{KO}$ ) $\left[F_{1,13}=23.56, P=0.0007\right]$ and a significant main effect for $\mathrm{T}$ cell type (WT versus KO) $\left[F_{1,13}=11.77, P=0.0064\right]$. The interaction between endothelial cell type and $\mathrm{T}$ cell type proved to be insignificant $\left[F_{1,13}=0.67, P=0.43\right]$. In transmigration assays using lung-derived endothelial cells, twoway ANOVA, with two between-group factors, also revealed a significant main effect for endothelial cell type (lung RC versus KO) $\left[F_{1,15}=27.39, P=0.0002\right]$ and a significant main effect for $\mathrm{T}$ cell type (WT versus KO) $\left[F_{1,15}=16.71, P=0.0015\right]$. The interaction between EC type and $\mathrm{T}$ cell type proved to be insignificant $\left[F_{1,15}=1.68, P=0.22\right]$.
Similar assays were performed in a second laboratory using PLP-specific T lymphoblasts isolated from PLP-immunized SJL mice transmigrating across these immortalized endothelioma cell lines. These endothelioma lines were also noted to express equivalent levels of ICAM-1, ICAM-2, and VCAM-1, but only WT cells express PECAM-1 (data not shown). Migration of these $T$ cells across PECAM-KO endotheliomas derived from brain (bEnd.PECAM-1.2), mesenteric lymph nodes (mlEnd.PECAM-1.1), and lung (luEnd.PECAM-1.1) was significantly increased in comparison with transmigration across WT endothelioma monolayers (bEnd.WT) (data not shown). Interestingly, while treatment of the endotheliomas with TNF- $\alpha$ increased surface expression of ICAM-1 and VCAM-1, it did not affect transmigration levels (data not shown) (44).

The differences in transmigration rates noted in Figures 3 and 4 likely reflect several differences in the endothelial cells and the $\mathrm{T}$ lymphocytes used. The endothelial cells used in Figure 4 were immortalized, cloned cells, whereas low-passage endothelial cells were used in Figure 3. Further, in other experiments T lymphoblasts isolated from PLP-immunized SJL mice were used, while the T lymphocytes used in Figures 3 and 4 were C57BL/6-derived MOG-specific T lymphocytes activated with MOG peptide and IL-2. These experiments, utilizing different endothelial cells and different $T$ lymphocyte populations, would be expected to yield disparate levels of transmigration. However, in all experiments performed, transmigration rate is dependent upon the presence or absence of endothelial PECAM-1. ICAM-1 levels were found to vary modestly on the cells. Increasing ICAM-1 and VCAM-1 expression by TNF- $\alpha$ treatment did not alter the transmigration rates, further supporting a role for endothelial PECAM-1 in this process.

Vascular permeability is increased in vitro and in vivo during development of EAE and dermal histamine challenge in PECAM-KO mice. Migration of leukocytes across endothelial cells has been shown to be enhanced by vasoactive substances that disrupt endothelial junctions and increase vascular permeability (52). We investigated whether endothelial junctional integrity may be compromised in PECAM-KO monolayers. Endothelioma cells derived from the lungs of PECAM-KO mice or the same cells reconstituted with PECAM-1 (PECAM-RC) were cultured to confluence on porous membranes as in the transmigration assays. In control samples, Evans blue dye was added to the upper chamber to confirm that the endothelial monolayers restricted its flow into the bottom chamber. Monolayers were treated with histamine, and at various time points later, dye was added. The dye that diffused into the lower wells was collected and measured spectrophotometrically (Figure 5). At 30 seconds after histamine exposure, dye flowed freely across either the PECAM-KO or PECAM-RC endothelial layers into the bottom chambers. Over the next 10-15 minutes the monolayers became increasingly impermeable to the dye. The rate 
a
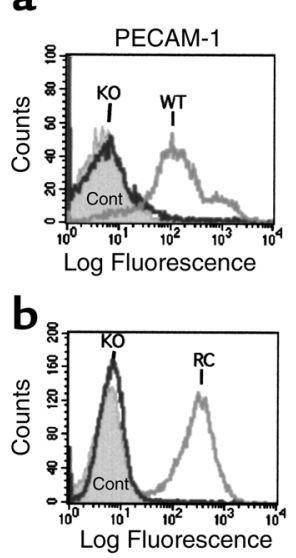

Log Fluorescence
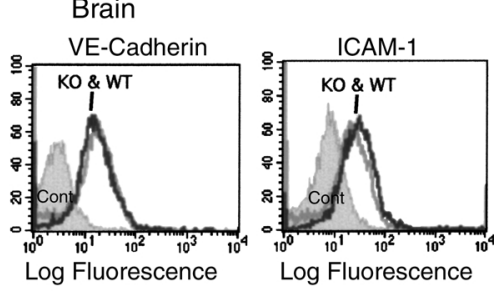

Lung
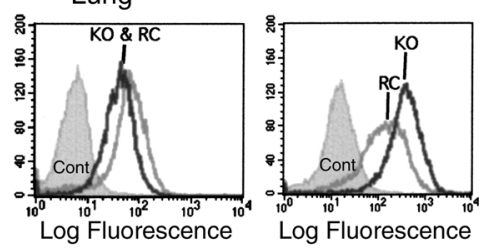

WT T Lymphocytes
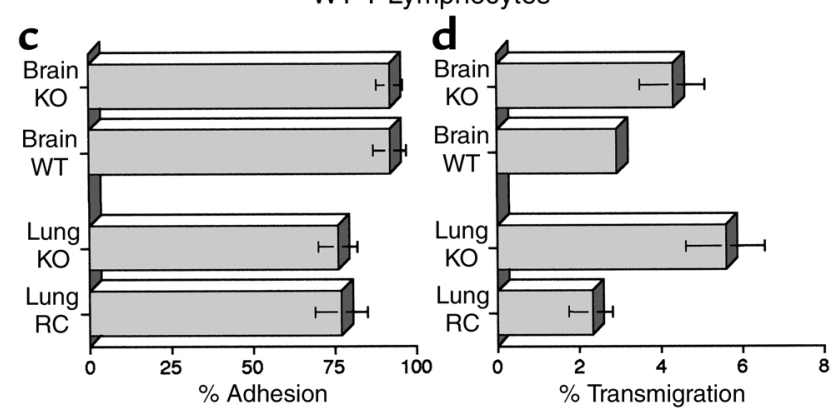

KO T Lymphocytes

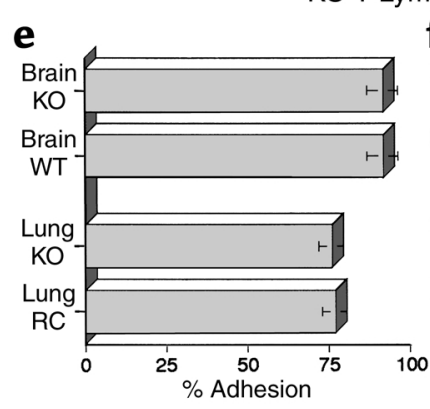

f

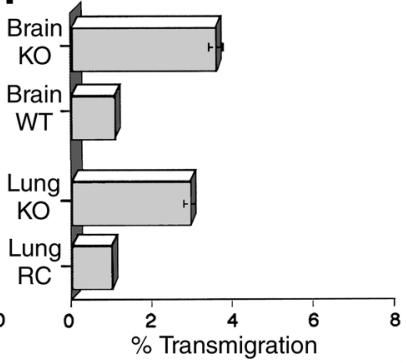

at which the endothelium became impermeable was faster for the PECAM-RC monolayer, and the barrier of these cells was completely restored by 15 minutes after histamine challenge. The PECAM-KO endothelial layer remained significantly permeable to the dye up to 20 minutes. The differences in the mean values among the groups ( $\mathrm{RC}$ and $\mathrm{KO}$ lung endothelia) are greater than

\section{Figure 4}

Immortalized endothelial cells derived from PECAM-KO mice support increased transmigration of MOG-specific T cells. ( $\mathbf{a}$ and $\mathbf{b}$ ) FACS analysis of immortalized endothelial cells derived from WT and $\mathrm{KO}$ brain (a) and KO lung and PECAM-reconstituted (RC) lung microvascular endothelial cells (b), illustrating the expression of VE-cadherin and ICAM- 1 in all four cell types, but PECAM- 1 expression only in the WT and PECAM-RC cells. (c and $\mathbf{e}$ ) In vitro adhesion assays illustrating no differences in MOG-specific $T$ cell adhesion to WT, PECAM-KO, and PECAM-RC endothelioma cells. ( $\mathbf{d}$ and $\mathbf{f}$ ) In vitro transmigration assays similar to those in Figure $2 c$ were performed using MOG-specific T cells transmigrating across monolayers of endothelioma cell lines. Transmigration of T cells across KO endotheliomas derived from brain and lung was consistently and significantly increased in comparison with transmigration across WT and PECAM-RC endotheliomas. In $\mathbf{c}$ and $\mathbf{d}$ WT T lymphocytes were used, and in $\mathbf{e}$ and $\mathbf{f}$ KO T lymphocytes were used. $n=4$.

would be expected by chance $\left(F=5.67, P<5.4 \times 10^{-5}\right)$. An all-pairwise multiple comparison procedure resulted in significant differences between the two groups from 2 through 15 minutes.

We next assessed the integrity of CNS vasculature in vivo using an Evans blue vital dye permeability assay. At various days after immunization with MOG peptide to induce EAE, dye was injected retro-orbitally and mice were perfused with PBS to clear it from the vasculature. The dye that had extravasated into the brain tissue was extracted and measured (Figure 6a). At early time points after immunization, the CNS vasculature of both WT and PECAM-KO mice was impermeable to dye. By day 6 , the vasculature became permeable to the dye, indicating a breach of integrity of the endothelial cell barrier. The brains of the PECAM-KO group exhibited a twofold higher average PI compared with the WT group, and there was a delay in the restoration of vascular integrity in PECAM-KO mice. The vasculature of WT mice became impermeable to the dye by day 12 , while the permeability barrier in the PECAM-KO mice was not restored until day 24 . The differences in the mean values among the groups (WT and KO mice) are greater than would be expected by chance $\left(F=17.9, P<2.9 \times 10^{-7}\right)$. An all-pairwise multiple comparison procedure resulted in significant differences between the two groups at days 6 and 12 . Skin vessel permeability was unaffected in both WT and PECAM-KO mice during the course of EAE, illustrating specificity of the response (Figure $6 \mathrm{~b})(F=0.196, P<0.82)$.

\section{Figure 5}

PECAM-KO endothelial cells exhibit prolonged permeability changes in response to histamine exposure in vitro. Lung-derived PECAM-KO microvascular endothelial cells and lung-derived PECAM-KO microvascular endothelial cells transfected with and expressing WT PECAM-1 (PECAM-RC) were cultured in confluent monolayers on transwell membranes, and exposed to histamine. The permeability of the monolayers to Evans blue dye was measured by collecting media from the lower wells and measuring the absorbance at $650 \mathrm{~nm}$. PECAM-KO endothelial cell monolayers (squares) exhibited prolonged permeability to the dye, lasting at least 20 minutes, while the PECAM-RC endothelial cell monolayers (diamonds) were impermeable to the dye by 15 minutes. $n=5$.

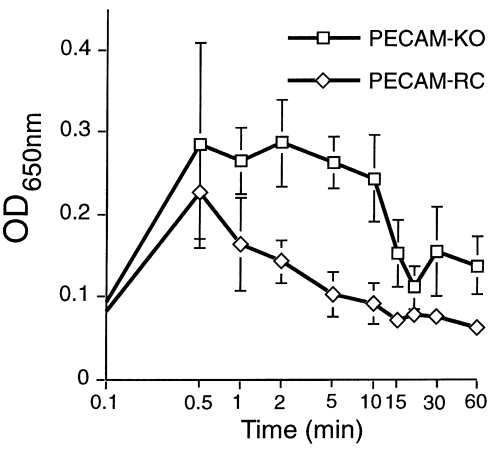



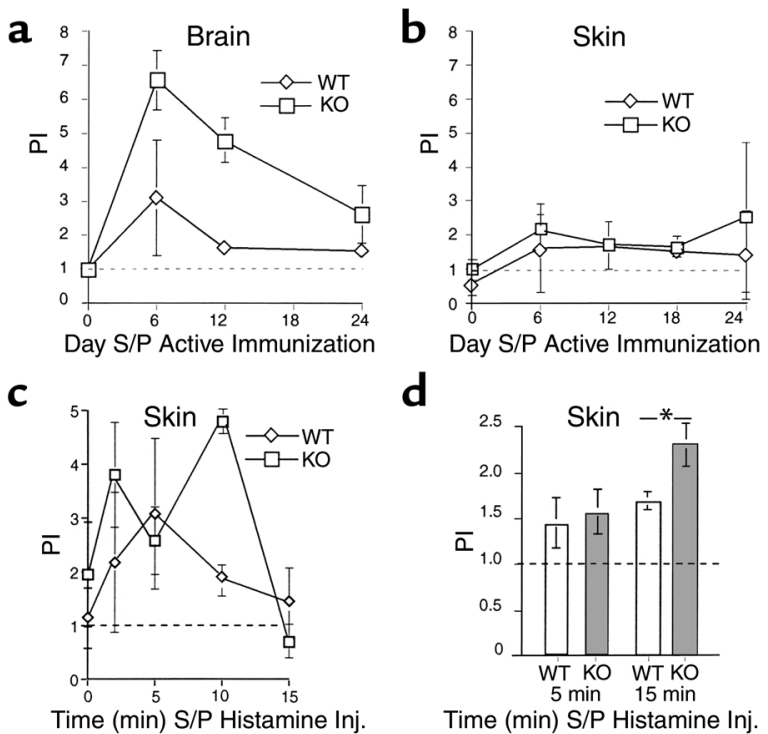

d

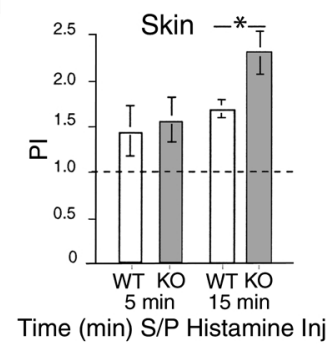

\section{Figure 6}

Challenged vessel permeability is altered in PECAM-KO mice. (a) PIs of mouse brain tissue after induction of EAE. In the PECAM-KO mice, there was a significant increase (twofold) in PI by day 6 after immunization, and the vessels remained permeable for a longer period of time compared with those of WT mice $(n=3, P<0.03)$. (b) Skin vessel PI was not affected during EAE in either group of mice $(n=3)$. (c) Pls in mouse skin following dermal histamine challenge. The vessels of the PECAM-KO mice remained markedly permeable to dye for up to 10 minutes after histamine injection, while the vessels of the WT mice are mostly impermeable to the dye by 10 minutes. Pls for both groups return to base line by 15 minutes after histamine injection $(n=3)$. (d) Due to the longer time period that the PECAM$K O$ vessels remain "leaky" following dermal histamine challenge, more dye accumulates in the skin tissue of the PECAM-KO mice by 15 minutes following histamine challenge than in that of WT mice $\left(n=3,{ }^{*} P=0.039\right) . \mathrm{S} / \mathrm{P}$, status post.

In a separate experiment, the skin vessels were challenged with histamine to increase dermal vascular permeability (Figure 6c). Dye was injected at various time points following intradermal injection of histamine or PBS, and extracted from the skin tissue. At early time points after histamine injection ( 0 minutes, 2 minutes, 5 minutes), dermal vascular permeability of both the WT and the PECAM-KO mice was increased and dynamic. However, by 10 minutes after histamine injection, the PECAM-KO skin vasculature remained markedly permeable to the dye, while the WT vasculature became impermeable. By 15 minutes, dermal vessel integrity had been restored in both groups of mice. The differences in the mean values among the groups (WT and $\mathrm{KO}$ mice) are greater than would be expected by chance $(F=2.86, P<0.044)$. An all-pairwise multiple comparison procedure resulted in significant differences between the two groups at 10 minutes.

In Figure 6d, dye was allowed to circulate in the blood for 45 minutes prior to vessel challenge with histamine so that dye accumulated in the tissue from the moment the junctions were compromised until the mice were sacrificed. Five minutes after histamine injection, equal amounts of dye had extravasated in both groups of mice. However, after 15 minutes an increase in the amount of dye extracted from the PECAM-KO mice indicates a greater accumulation of extravasated dye in this group. This result is consistent with the previous experiment, in that the prolonged time required for restoration of vascular integrity in the PECAM-KO mice would result in a longer period of vascular permeability, leading to greater accumulation of dye in the skin tissue. The dermal vessel experiments confirm the hypothesis that the restoration of vascular integrity is impaired in the PECAM-KO mice, which could lead to an exacerbated inflammatory response.

\section{Discussion}

These data suggest an unexpected proinflammatory phenotype in PECAM-KO mice during the progression of EAE, illustrated by increased immune cell trafficking into the CNS during early disease and early onset of clinical symptoms. During EAE, peripheral blood lymphocytes must adhere to and migrate across the endothelial blood-brain barrier that normally restricts their entry into the CNS. During inflammation, the vessels become permeable to soluble inflammatory mediators, antigen-specific $\mathrm{T}$ cells, and recruited inflammatory cells. In PECAM-KO mice, re-equilibration of the blood-brain barrier was delayed, which may account for the increased ability of leukocytes to migrate across the blood-brain barrier.

In vitro, PECAM-KO endothelial cells supported enhanced transendothelial migration of $\mathrm{T}$ cells. This result may seem counterintuitive, given that several reports indicate that PECAM-1 is an important contributor to leukocyte transendothelial migration in vitro (13-15) and in vivo (16-19). However, published data show that the requirement for PECAM-1 in the transmigration of leukocytes is not absolute. Particularly, PECAM-KO mice demonstrate a transient arrest, but not a reduction, of transendothelial migration of neutrophils in a peritoneal inflammation model. The authors of that report and others suggest that there may be compensation by other adhesion molecules in the absence of PECAM-1 (21).

Several technical differences may also explain these apparent discrepancies. First, there are inherent differences between antibody-blocking experiments and those carried out using PECAM-KO mice or cells. Furthermore, most previous studies have examined in vitro transmigration or in vivo models of inflammation mediated by neutrophils or monocytes, while EAE is initiated by antigen-specific $T$ cells. It has been reported that a $T$ cell population that has been depleted of PECAM- $1^{+}$cells is able to transmigrate normally (20). In addition, PECAM- 1 expression varies among different $\mathrm{T}$ cell subsets, and therefore the requirement for PECAM-1 may differ between the wide variety of $\mathrm{T}$ cell subsets (naive, activated, memory, $\mathrm{CD}^{+}, \mathrm{CD}^{+}$). It is also important to consider the acute or chronic nature of the inflammatory response studied in the many in 
vivo models. Most studies of the role of PECAM-1 in inflammation examine in vivo models that measure leukocyte transmigration over a short period of time (minutes to hours), during an acute response. Although initial trafficking of T cells into the CNS during EAE may occur within hours, significant extravasation into the CNS tissue occurs over days, and disease progresses over weeks. The availability of the PECAMKO mouse will allow for continued assessment of inflammation and cellular behavior in the complete absence of PECAM-1.

We hypothesize a role for PECAM-1 in the restoration of interendothelial junctional integrity following disrupting stimuli, while normal, homeostatic endothelium is not affected (Figures 5 and $6 \mathrm{~b}$ ). The prolonged vascular permeability in the PECAM-KO mice could be accounted for if PECAM-1 plays a role in re-establishing intact junctions following inflammatory stimuli and transendothelial migration of leukocytes. In turn, disruption of endothelial cell-cell junctions has been shown to facilitate transmigration of leukocytes (52), which may contribute to a cycle of increased migration and decreased junctional integrity in the PECAM-KO mice. However, in each of our experiments, the endothelial permeability barrier was eventually restored, showing that the requirement for PECAM-1 at interendothelial junctions is not absolute.

Prior studies provide support for the significance of PECAM-1 at the endothelial cell-cell junctions. PECAM- 1 is concentrated at cell contact sites in all continuous endothelial cell linings in vivo (2) and is proximal to adherens junctions (53). Anti-PECAM- 1 antibodies have been shown to prevent endothelial cells from developing intercellular contacts and achieving confluence in vitro (3) and to attenuate angiogenesis in vivo and endothelial cell tube formation in vitro (54).

Endothelial cell-cell junctions are complex structures consisting of transmembrane proteins and a group of submembranous proteins that link to the actin-based cytoskeleton (55). $\beta$-Catenin, which normally associates with the transmembrane junctional protein VE-cadherin, linking it to cytoskeletal proteins, was also found to associate with PECAM-1 (54). When stimuli, such as VEGF stimulation, induce junctional disassembly, $\beta$-catenin becomes a target of kinases (56). Tyrosinephosphorylated $\beta$-catenin dissociates from VE-cadherin and associates with PECAM-1 (29). The presence of phosphorylated $\beta$-catenin and protein tyrosine phosphatases such as SHP-2 on the same scaffolding molecule may induce the dephosphorylation of $\beta$-catenin, allowing it to reassociate with VE-cadherin, thus reassembling intact adherens junctional complexes (29). Perhaps in the PECAM-KO mice, PECAM-1 cannot act as a reservoir for phosphorylated $\beta$-catenin, resulting in increased cytoplasmic pools of the phosphorylated protein, which cannot readily reassociate with VE-cadherin in the junctional complex. Ultimately, $\beta$-catenin may become dephosphorylated by other mechanisms and resume its association with
VE-cadherin and other junctional proteins, thus eventually restoring integrity of the endothelial cell layer. Further elucidation of the underlying mechanism(s) by which PECAM-1 modulates vascular integrity will aid in our understanding of the roles PECAM-1 plays during inflammatory responses. Although we have not discounted the possibility that signals mediated via PECAM-1 may contribute toward the generation or propagation of the immune response during EAE, the impairment of dynamic vascular junctional integrity is likely to be one of the driving forces in the early EAE phenotype of the PECAM-KO mice.

\section{Acknowledgments}

This work was supported in part by US Public Health Service grants R37-HL-28737, PPG DK-38979, and RO1-HL-51018 to J.A. Madri; National Multiple Sclerosis Society grant RG 2394 to N.H. Ruddle; and a Richard K. Gershon fellowship to A. Juedes.

1. Newman, P., et al. 1990. PECAM-1 (CD31) cloning and relation to adhesion molecules of the immunoglobulin gene superfamily. Science. 24:1219-1222.

2.Muller, W., Ratti, C., McDonnell, S., and Cohn, Z. 1989. A human endothelial cell-restricted, externally disposed plasmolemmal protein enriched in intercellular junctions. J. Exp. Med. 170:399-414.

3. Albelda, S., Oliver, P., Romer, L., and Buck, C. 1990. EndoCAM: a novel endothelial cell-cell adhesion molecule. J. Cell Biol. 110:1227-1237.

4. Ohto, H., et al. 1985. A novel leukocyte differentiation antigen: two monoclonal antibodies TM2 and TM3 define a 120-kd molecule present on neutrophils, monocytes, platelets, and activated lymphoblasts. Blood. 66:873-881.

5. Goyert, S., et al. 1986. Biochemistry and expression of myelomonocytic antigens. J. Immunol. 137:3909-3914.

6. Stockinger, S., et al. 1990. Molecular characterization and functional analysis of the leukocyte surface protein CD31. J. Immunol. 145:3889-3897.

7. Ashman, L., and Aylett, G. 1991. Expression of CD31 epitopes on human lymphocytes: CD31 monoclonal antibodies differentiate between naive (CD45RA + ) and memory (CD45RA-) CD4-positive T cells. Tissue Antigens. 38:208-212.

8. Albelda, S., Muller, W., Buck, C., and Newman, P. 1991. Molecular and cellular properties of PECAM-1 (endoCAM/CD31): a novel vascular cellcell adhesion molecule. J. Cell Biol. 114:1059-1068.

9. Xie, Y., and Muller, W. 1993. Molecular cloning and adhesive properties of murine platelet/endothelial cell adhesion molecule 1. Proc. Natl. Acad. Sci. USA. 90:5569-5573.

10. Fawcett, J., et al. 1995. Mapping the homotypic binding sites in CD31 and the role of CD31 adhesion in the formation of interendothelial cell contacts. J. Cell Biol. 128:1229-1241.

11. Muller, W., Berman, M., Newman, P., DeLisser, H., and Albelda, S. 1992. A heterophilic adhesion mechanism for platelet/endothelial cell adhesion molecule-1. J. Exp. Med. 175:1401-1404.

12. DeLisser, H., et al. 1993. Platelet/endothelial cell adhesion molecule-1 (CD31)-mediated cellular aggregation involves surface glycosaminoglycans. J. Biol. Chem. 268:16037-16046.

13. Muller, W., Weigl, S., Deng, X., and Phillips, D. 1993. PECAM-1 is required for transendothelial migration of leukocytes. J. Exp. Med. 178:449-460.

14. Liao, F., et al. 1995. Migration of monocytes across endothelium and passage through extracellular matrix involve separate molecular domains of PECAM-1. J. Exp. Med. 182:1337-1343.

15. Berman, M., Xie, Y., and Muller, W. 1996. Roles of platelet/endothelial cell adhesion molecule-1 (PECAM-1/CD31) in natural killer cell transendothelial cell migration and beta 2 integrin activation. J. Immunol. 156:1515-1524.

16. Vaporciyan, A., et al. 1993. Involvement of platelet/endothelial cell adhesion molecule-1 in neutrophil recruitment in vivo. Science. 262:1580-1582.

17. Bogen, S., Pak, J., Garifallou, M., Deng, X., and Muller, W. 1994. Monoclonal antibody to murine PECAM-1 (CD31) blocks acute inflammation in vivo. J. Exp. Med. 179:1059-1061.

18. Wakelin, M., et al. 1996. An anti-platelet-endothelial cell adhesion molecule-1 antibody inhibits leukocyte extravasation from mesenteric 
microvessels in vivo by blocking the passage through the basement membrane. J. Exp. Med. 184:229-239.

19. Nakada, M., et al. 2000. Antibodies against the first Ig-like domain of human platelet endothelial cell adhesion molecule-1 (PECAM-1) that inhibit PECAM-1 dependent homophilic adhesion block in vivo neutrophil recruitment. J. Immunol. 164:452-462.

20. Bird, I., Spragg, J., Ager, A., and Matthews, N. 1993. Studies of lymphocyte transendothelial migration: analysis of migrated cell phenotypes with regard to CD31 (PECAM-1), CD45RA and CD45RO. Immunology. 80:553-560.

21. Duncan, G., et al. 1999. Genetic evidence for functional redundancy of platelet/endothelial cell adhesion molecule-1 (PECAM-1): CD31-deficient mice reveal PECAM-1-dependent and PECAM-1-independent functions. J. Immunol. 162:3022-3030.

22. Newman, P., et al. 1992. Activation-dependent changes in human platelet PECAM-1 phosphorylation, cytoskeletal association, and surface membrane redistribution. J. Cell Biol. 119:239-246.

23. Zehnder, J., et al. 1992. The cell adhesion molecule CD31 is phosphorylated after cell activation. J. Biol. Chem. 267:5243-5249.

24. Lu, T., Yan, L., and Madri, J. 1996. Integrin engagement mediates tyrosine dephosphorylation on platelet-endothelial cell adhesion molecule 1. Proc. Natl. Acad. Sci. USA. 93:11808-11813.

25. Sagawa, K., Swaim, W., Zhang, J., Unsworth, E., and Siraganian, R. 1997. Aggregation of high affinity IgE receptor results in the tyrosine phosphorylation of the surface adhesion protein PECAM-1 (CD31). J. Biol. Chem. 272:13412-13418.

26. Osawa, M., Masuda, M., Harada, N., Lopes, R., and Fujiwara, K. 1997. Tyrosine phosphorylation of platelet endothelial cell adhesion molecule1 (PECAM-1, CD31) in mechanically stimulated vascular endothelial cells. Eur. J. Cell Biol. 72:229-237.

27. Jackson, D., Ward, C., Wang, R., and Newman, P. 1997. The protein-tyrosine phosphatase SHP-2 binds platelet/endothelial cell adhesion molecule-1 (PECAM-1) and forms a distinct signaling complex during platelet aggregation. J. Biol. Chem. 272:6986-6993.

28. Sagawa, K., Kimura, T., Swieter, M., and Siraganian, R. 1997. The protein-tyrosine phosphatase SHP-2 associates with tyrosine-phosphorylated adhesion molecule PECAM-1 (CD31). J. Biol. Chem. 272:31086-31091.

29. Ilan, N., Mahooti, S., Rimm, D., and Madri, J. 1999. PECAM-1 (CD31) functions as a reservoir and modulator of tyrosine-phosphorylated $\beta$-catenin. J. Cell Sci. 112:3005-3014.

30. Lu, T., Barreuther, M., Davis, S., and Madri, J. 1997. Platelet endothelia cell adhesion molecule- 1 is phosphorylatable by c-Src, binds Src-Src homology 2 domain, and exhibits immunoreceptor tyrosine-based activation motif-like properties. J. Biol. Chem. 272:14442-14446.

31. Tanaka, Y., et al. 1992. CD31 expressed on distinctive T cell subsets is a preferential amplifier of B1 integrin-mediated adhesion. J. Exp. Med. 176:245-253.

32. Piali, L., et al. 1993. Murine platelet endothelial cell adhesion molecule (PECAM-1)/CD31 modulates beta2 integrins on lymphokine-activated killer cells. Eur. J. Immunol. 23:2464-2471.

33. Poggi, A., Panzeri, M., Moretta, L., and Zocchi, M. 1996. CD31-triggered rearrangement of the actin cytoskeleton in human natural killer cells. Eur. J. Immunol. 26:817-824.

34. Elias, C., et al. 1998. Ligation of CD31/PECAM-1 modulates the function of lymphocytes, monocytes and neutrophils. Eur. J. Immunol. 28:1948-1958.

35. Hynes, R. 1992. Integrins: versatility, modulation, and signaling in cell adhesion. Cell. 60:11-15.

36. Newton-Nash, D., and Newman, P. 1999. A new role for plateletendothelial cell adhesion molecule-1 (CD31): inhibition of TCR-mediated signal transduction. J. Immunol. 163:682-688.

37. Newman, D., Hamilton, C., and Newman, P. 2001. Inhibition of antigenreceptor signaling by platelet-endothelial cell adhesion molecule-1
(CD31) requires functional ITIMs, SHP-2, and p56lck. Blood. 97:2351-2357.

38. Swanborg, R. 1995. Experimental autoimmune encephalomyelitis in rodents as a model for human demyelinating disease. Clin. Immunol. 77:4-13.

39. Williams, K.C., Zhou, R.W., Ueno, K., and Hickey, W.F. 1996. PECAM-1 (CD31) expression in the central nervous system and its role in experimental allergic encephalomyelitis in the rat. J. Neurosci. Res. 45:747-757.

40. Mendel, I., Kerlero de Rosbo, N., and Ben-Nun, A. 1995. A myelin oligodendrocyte glycoprotein peptide induces typical chronic experimental autoimmune encephalomyelitis in $\mathrm{H}-2 \mathrm{~b}$ mice: fine specificity and $\mathrm{T}$ cell receptor $\mathrm{V} \beta$ expression of encephalitogenic T cells. Eur. J. Immunol. 25:1951-1959.

41. Plendl, J., Hartwell, L., and Auerbach, R. 1993. Organ-specific change in Dolichos biflorus lectin binding by myocardial endothelial cells during in vitro cultivation. In Vitro Cell. Dev. Biol. 29A:25-31.

42. Laschinger, M., and Engelhardt, B. 2000. Interaction of alpha4-integrin with VCAM-1 is involved in adhesion of encephalitogenic T cell blasts to brain endothelium but not in their transendothelial migration in vitro. J. Neuroimmunol. 102:32-43.

43. Engelhardt, B., et al. 1998. The development of experimental autoimmune encephalomyelitis in the mouse requires alpha4-integrin but not alpha4beta7-integrin. J. Clin. Invest. 102:2096-2105.

44. Reiss, Y., Hoch, G., Deutsch, U., and Engelhardt, B. 1998. T cell interaction with ICAM-1 deficient endothelium in vitro: requisite role for ICAM-1 and ICAM-2 in transendothelial migration of T cells. Eur. J. Immunol. 28:3086-3099.

45. Wong, C., et al. 2000. PECAM-1/CD31 trans-homophilic binding at the intercellular junctions is independent of its cytoplasmic domain; evidence for heterophilic interaction with integrin $\alpha v \beta 3$ in cis. Mol. Biol. Cell. 11:3109-3121.

46. Morgenstern, J., and Land, H. 1990. Advanced mammalian gene transfer high titer retroviral vectors with multiple drug selection markers and a complementary help-free packaging cell line. Nucleic Acids Res. 18:3587-3596

47. Mahooti, S., et al. 2000. PECAM-1 (CD31) expression modulates bleeding time in vivo. Am. J. Pathol. 157:75-81.

48. Graesser, D., et al. 1998. The inter-relationship of alpha4 integrin and matrix metalloproteinase- 2 in the pathogenesis of experimental autoimmune encephalomyelitis. Lab. Invest. 78:1445-1458.

49. Graesser, D., Mahooti, S., and Madri, J. 2000. Distinct roles for matrix metalloproteinase-1 and alpha4 integrin in autoimmune $\mathrm{T}$ cell extravasation and residency in brain parenchyma during experimental autoimmune encephalomyelitis. J. Neuroimmunol. 109:121-131.

50. Gijbels, K., Galardy, R., and Steinman, L. 1994. Reversal of experimental autoimmune encephalomyelitis with a hydroxymate inhibitor of matrix metalloproteinases. J. Clin. Invest. 94:2177-2182.

51. Eliceiri, B., et al. 1999. Selective requirement for src kinases during VEGFinduced angiogenesis and vascular permeability. Mol. Cell. 4:915-924.

52. Singhal, P., Sankaran, R., Nahar, N., Shah, N., and Patel, P. 2000. Vasoactive agents modulate migration of monocytes across glomerular endothelial cells. J. Investig. Med. 48:110-117.

53. Ayalan, O., Sabanai, H., Lampugnani, M., Dejana, E., and Geiger, B. 1994 Spatial and temporal relationships between cadherins and PECAM-1 in cell-cell junctions of human endothelial cells. J. Cell Biol. 126:247-258.

54. Matsumura, T., Wolff, K., and Petzelbauer, P. 1997. Endothelial cell tube formation depends on cadherin 5 and CD31 interactions with filamentous actin. J. Immunol. 158:3408-3416.

55. Geiger, B., Ginsberg, D., Salomon, D., and Volberg, T. 1991. The molecular basis for the assembly and modulation of adherens-type junctions. Cell Differ. Dev. 32:343-353.

56. Esser, S., Lampugnani, M., Corada, M., Dejana, E., and Risau, W. 1998. Vascular endothelial growth factor induces VE-cadherin tyrosine phosphorylation in endothelial cells. J. Cell Sci. 111:1853-1865. 